\title{
Is the stellar initial mass function universal?
}

\author{
Ignacio Ferreras $^{1 *}$, Francesco La Barbera ${ }^{2}$, Alexandre Vazdekis ${ }^{3,4}$ \\ ${ }^{1}$ Mullard Space Science Laboratory, University College London, Dorking, Surrey RH5 6NT, UK \\ ${ }^{2}$ INAF-Osservatorio Astronomico di Capodimonte, I-80131 Napoli, Italy \\ ${ }^{3}$ Instituto de Astrofísica de Canarias, E-38200 La Laguna, Tenerife, Spain \\ ${ }^{4}$ Departamento de Astrofísica, Universidad de La Laguna, E-38205 La Laguna, Tenerife, Spain \\ *E-mail: i.ferreras@ucl.ac.uk
}

Astronomy \& Geophysics, vol.57, April 2016

\begin{abstract}
The most essential components of a galaxy are the dark matter halo and the baryonic material, the latter mostly made up of gas and stars. The physical processes that transform gas into stars and vice-versa are one of the most complex, unsolved challenges in astrophysics, and the stellar initial mass function (IMF) represents a fundamental piece in this entangled cosmic puzzle. The IMF is the distribution of stellar masses in a star forming region. It allows us to create synthetic models of the UV/Optical/IR spectral energy distribution of galaxies by combining our knowledge of stellar evolution with the adequate proportion of massive, intermediate and low-mass stars. A change in the IMF introduces drastic effects both on galactic chemical enrichment - the build up of chemical elements as the galaxy evolves - and on the interpretation of observables such as NUV and $\mathrm{H} \alpha$ fluxes into star formation rates. It is also essential for the estimation of stellar masses from photometry. Moreover, a change in the IMF affects the gas mass budget between different generations of stars, hence modifying the characteristic formation timescales of galaxies. In this article, we review the recent advances in the field, led by observations that suggest a significant departure from the Milky Way standard in the most massive early-type galaxies.
\end{abstract}

The initial mass function is the distribution of stellar masses at birth in a stellar population. It constitutes one of the key ingredients when building up the stellar populations in a galaxy. The IMF controls the relative contribution from massive, intermediate and low-mass stars. Therefore, most physical quantities derived from observables depend sensitively on the choice of IMF, such as the conversion from NUV/emission line fluxes into star formation rates; the derivation of stellar masses from photometry; or the evolution of chemical composition within a galaxy (galactic chemical enrichment). The IMF has been traditionally constrained by measurements in nearby regions, mostly targeting resolved stellar populations (i.e. star clusters and $\mathrm{OB}$ associations) in the Milky Way galaxy. These studies produced the standard functions we commonly use, such as the original IMF defined by Salpeter (1955); or more detailed ones (e.g. Miller-Scalo, Kroupa, Chabrier, see Kroupa et al., 2013, for a recent review). Constraining the IMF in other galaxies involves the study of non-resolved stellar populations, where light originates from large ensembles of stars involving a wide range of masses, chemical composition, age and evolutionary phases. The seminal studies of Spinrad in the 1970s explored the possibility of constraining the IMF through spectroscopic data targeting the differential effect of giant and dwarf stars on selected absorption features. Over the past decades, a number of papers showed inconclusive results, mostly due to the lack of proper instrumentation and population synthesis models. This article focuses on results recently published in this field, that reject the hypothesis of a universal stellar IMF in massive early-type galaxies. It is split into four sections, presenting stellar population synthesis models; the interpretation of spectral absorption 
features; additional probes of the IMF, finishing with a section on the implications of IMF variations on the formation process of galaxies.

\section{Population Synthesis Modelling}

The essential tool to constrain the stellar content in external galaxies - where individual stars cannot be resolved - is the stellar population synthesis model (see, e.g. Tinsley \& Gunn, 1976; Bruzual \& Charlot, 2003; Vazdekis et al., 2012). These models combine i) our current knowledge of stellar evolution covering all stellar spectral types with ii) extensive stellar spectral libraries, either theoretical or empirical, and iii) a prescription for the stellar Initial Mass Function (IMF). The method for obtaining relevant stellar population parameters consists of matching the observed colours and absorption line index measurements of galaxies with the predictions from these models. However, the derivation of star formation histories from the photometric and spectroscopic data is complicated by a well-known degeneracy between age and metallicity (e.g. Worthey, 1994). Therefore, this method is especially suitable for the populations found in early-type galaxies (hereafter ETGs). These galaxies have a relatively homogeneous stellar component, and the contribution from gas and dust to the spectral energy distribution in the optical is minor. Much progress has been made during the last decade to lift the agemetallicity degeneracy with improved models including spectroscopic stellar libraries at higher resolution, for which the contribution to the absorption lines from different parameters of the populations can be constrained in more detail.

One of the major achievements of population synthesis in the analysis of galaxy formation has been the interpretation of age- and metallicity-sensitive spectral indices in ETGs. A strong correlation exists between the mass of an ETG and their spectral indices based on the Hydrogen Balmer series, or on metal-dependent indices sensitive to Magnesium. In contrast, Iron-sensitive indices do not follow this trend (Trager et al., 2000). Population synthesis models helped to interpret these results as a trend in stellar ages and metallicity, such that the most massive ETGs form their populations very early, over a remarkably short period of time $(\lesssim 1 \mathrm{Gyr})$, whereas lower-mass ETGs have a more extended process of star formation, possibly ejecting considerable amounts of metals in outflows (e.g. Ferreras \& Silk, 2000; Thomas et al., 2005). Furthermore, environmentrelated differences in the stellar populations of ETGs have been found, with a significant truncation in the star formation history of satellite galaxies - those that fall into the dark matter halo of a larger structure (La Barbera et al., 2014). In general, constraints from population synthesis have shed light on the connection between structure growth - driven by the assembly of dark matter halos - and the so-called baryon physics that drives the primordial gas into stars at the centres of these halos. In fact, population synthesis constraints have been essential in defining the detailed prescriptions related to star formation and feedback in large numerical simulations of galaxy formation (e.g. De Lucia et al., 2006).

Great efforts are being devoted to enhance population synthesis models on several fronts. On stellar evolution modelling, for instance, the importance of the thermally-pulsing AGB phase is still debated (Maraston, 2005). Such a phase is especially prominent in $\sim 1 \mathrm{Gyr}-$ old populations, and will introduce an important systematic if not treated properly. Regarding stellar libraries, it is worth emphasizing that all population synthesis models at present rely on a relatively small grid of stellar spectra. Theoretical models of stellar atmospheres are still not ideal to provide a standalone grid of spectral libraries. They can be rather unreliable especially towards lower surface temperatures, where atomic and molecular absorption lines are notably hard to model. On the other hand, empirical libraries require a very delicate observational work, resulting in a relatively small number of stellar templates (for instance, $\sim 1,000$ stellar spectra for the MILES library, Sánchez-Blázquez et al. 2006). With such numbers of reference stars, one should be aware that some specific phase on the Hertzsprung-Russell diagram could be only modelled by a handful of stars in some cases. In addition to age, chemical composition can be described simplistically by an overall metallicity, defined as the mass fraction in elements other than hydrogen or helium. However, this approach implies a fixed abundance ratio among heavy elements, usually fixed to the solar reference. Non-solar abundance ratios are becoming increasingly important in detailed analyses of galaxy spectra, and such treatment often relies on models that combine empirical libraries and theoretical models (e.g. Conroy \& van Dokkum, 2012a), although empirical models begin to provide alternative routes to this problem (Vazdekis et al., 2015). Moreover, the ef- 
fect of non-solar abundances requires a consistent treatment of the stellar evolutionary tracks (Pietrinferni et al., 2006).

\section{IMF constraints from spectral features}

In the neighbourhood of the solar system and in stellar associations of our Galaxy (e.g. globular clusters), constraints on the IMF can be derived through direct star counts. In contrast, knowledge of the stellar IMF in distant galaxies (i.e. unresolved stellar populations) relies on indirect methods. Since the pioneering efforts of Spinrad (1962), it was realized that the stellar IMF of "unresolved" stellar populations can be constrained through specific spectral features, with prominent sensitivity to the surface gravity of stars (i.e. dwarf vs giant stars). These gravity-sensitive features include molecular bands (e.g. $\mathrm{TiO}, \mathrm{CaH}, \mathrm{FeH}$ ) and atomic lines (e.g. $\mathrm{NaI}, \mathrm{CaI}, \mathrm{CaII})$, that are either prominent in the spectra of dwarf stars (e.g. the NaI doublet at $\lambda \sim 8190 \AA$, and the Wing-Ford band, $\lambda \sim 9916 \AA$ ), or barely seen in dwarfs and most prominent in giants (e.g. the CaII NIR triplet). Figure 1 shows schematically how gravitysensitive spectral indices can be used to constrain the presence of low-mass and intermediate mass stars in an old population.

Unfortunately, this kind of analysis is far from trivial. The absorption lines found in the spectra of galaxies is a result of the superposition of an enormous number of stars over a range of ages, and chemical composition. Furthermore, the relative motion of these stars, characterized by a velocity dispersion, produces an even more complex blend because of the Doppler effect. Therefore, the interpretation of an absorption line in the spectrum of a galaxy is a complex task (see Figure 2). In addition, most gravity-sensitive features are located redwards of $6000 \AA$, where atmospheric absorption and emission lines strongly contaminate the spectrum of a galaxy. Moreover, changes in the equivalent width of the spectral feature related to IMF variations represent a small fraction of the fiducial line strength. For instance, the IMF-sensitive NaI8190 feature produces a $\sim 5 \%$ absorption in the spectrum of a massive ETG, but at redshift $\mathrm{z} \sim 0$, it overlaps almost exactly with a band of strong (20-50\%) telluric absorption lines from water vapour. In the 1970s and 1980s, discordant results were reported about dwarf stars contributing from a few percent to a significant fraction (up to 40\%) of the integrated light of ETGs (e.g. Spinrad \& Taylor, 1971). This contribution from cold (M-type) dwarfs to the integrated galaxy light would imply that a large fraction of the total mass budget of a galaxy should be in the form of M-dwarfs, increasing the stellar mass-to-light ratio to thirty or more. Interestingly, these first results appeared almost at the same time as dark-matter models of structure formation were establishing themselves as the reference hierarchical paradigm of galaxy formation. Nowadays, galaxy dynamics and strong lensing confine the mass-lo-light ratio of massive galaxies to values below ten, implying that M-dwarfs can only contribute a few percent to the integrated galaxy light. Albeit small, this contribution can change the estimated stellar mass of a galaxy by a factor of two or more, and has significant implications for the chemical enrichment history of a galaxy (which is driven by the ejected material of exploding supernovae from high-mass stars), and thus, ultimately, for our understanding of galaxy formation and evolution. However, those early results were inconclusive, and the interest in these gravity-sensitive features fizzled out.

At the turn of the millennium, improvements in population synthesis models and detector technology - increasing the efficiency of spectrographs in the red/NIR spectral window - allowed for a revision of the early attempts at constraining the IMF of unresolved populations through gravity-sensitive features. In Saglia et al. (2002) and Cenarro et al. (2003), it was found that while most metallic lines were stronger in the most massive ETGs, the NIR calcium triplet (CaT) showed the opposite behaviour. This trend could be either interpreted as a depletion of $\mathrm{Ca}$ in massive galaxies, or as an increased contribution of dwarf stars, thus reopening the case for a non-universal IMF, whereby massive ETGs feature a bottom-heavy mass function, i.e. an excess of low-mass stars with respect to the Milky-Way standard. Over the past few-years, a number of papers have added evidence in favour of this trend, with additional features such as the NaI doublet at $8190 \AA$ and the FeH-based Wing-Ford band, reaching measurements at sub-percent levels (van Dokkum \& Conroy, 2010), i.e. well below the variation expected from a few percent contribution of M-dwarfs to the total luminosity of the galaxy. More recently, our group showed that gravity-sensitive features can be studied with massive spectroscopic datasets, such as the one provided by the Sloan Digital Sky Survey (SDSS). By stacking thousands of spectra it is possible 
to achieve signal-to-noise ratios up to several hundreds per Angstrom. Furthermore, one can build stacks from galaxies at different redshift (where telluric lines contaminate different rest-frame spectral regions), so that gravity-sensitive features can be measured with an accuracy better than $0.1 \%$ (La Barbera et al., 2013).

The main challenge currently faced by these studies is the degeneracy between IMF and other population parameters, in particular elemental abundance variations. Changes in the line strengths of IMF-sensitive features can be simply produced by changing the individual elements causing them. Note that the star formation histories of massive ETGs are substantially different from that of our Milky Way galaxy. Therefore, departures of elemental abundances with respect to the solar values are to be expected. To break this IMF-abundance degeneracy, we need to combine several abundance- and gravitysensitive lines, carefully comparing their strengths to predictions of stellar population models with a range of non-solar abundance ratios. While such models are starting to appear, they rely mostly on theoretical predictions, and thus are unavoidably affected by uncertainties in modelling stellar evolution, atmospheres, etc. In this regard, the models of Conroy \& van Dokkum (2012a), based on state-of-art theoretical stellar population models, have provided a significant leap forward in our understanding of the stellar IMF. Using a full spectral fitting code - where one fits the spectral energy distribution over a wide range of wavelengths, rather than the strengths of individual lines - they obtained evidence towards a systematic variation of the IMF with velocity dispersion in ETGs (Conroy \& van Dokkum, 2012b). At the same time, our group (Ferreras et al., 2013; La Barbera et al., 2013) applied a comprehensive set of gravitysensitive features to test the universality of the IMF (Figure 3 illustrates the result with three grids targeting several spectral indices that depend in different ways on the population parameters). Analyzing $\mathrm{Ca}, \mathrm{Na}, \mathrm{Mg}$, and $\mathrm{TiO}$ features simultaneously, for thousands of spectra from the SDSS, we found a systematic increase of the dwarfto-giant ratio with velocity dispersion for the whole population of ETGs. An empirical method was applied only based on observed trends from the data - to correct for non-solar abundance ratios, minimizing the use of theoretical model predictions. Both methods gave a similar trend towards a bottom-heavy IMF in massive ETGs (see also Spiniello et al., 2015). Notwithstanding all the underlying difficulties, it is remarkable that at the mo- ment, no study of gravity-sensitive features in ETGs has been able to explain the observed trends without invoking a varying, non-universal, IMF.

In order to understand the origin of a non-universal IMF in ETGs, the next steps aim at probing local variations, i.e. within galaxies. Several recent studies based on long-slit spectra and integral field unit data have shown that variations in the IMF exist locally, with a bottom-heavy distribution in the central regions, where the populations are metal-rich, and have high velocity dispersion (Martín-Navarro et al., 2015a). Furthermore, metallicity appears to show the highest correlation with the IMF in these regions (Martín-Navarro et al., 2015b), although more work needs to be done to disentangle the observed trends with respect to individual element abundances.

\section{Orthogonal probes of the IMF}

In addition to methods targeting spectroscopic signatures of giant and dwarf stars, it is possible to constrain the IMF, indirectly, through the stellar mass-to-light ratio $(\mathrm{M} / \mathrm{L})$. In this case, good photometry and an accurate determination of the stellar mass of a galaxy are needed. Two main techniques can be used: direct dynamical tracers, and strong gravitational lensing. Note that dynamical and lensing methods will be sensitive to all gravitational material. Therefore, the presence of gas and, most importantly, dark matter, has to be accounted for. Therefore, the best possible targets for these studies are, once more, the central regions of ETGs, where the gas or dust content is minimal, and the presence of dark matter should be subdominant. Dynamics-based methods aim at fitting the observed kinematics (i.e. the velocity rotation and velocity dispersion profiles of the stars) with a gravitational potential. Typically, some variation of Jeans' equations is applied. The analysis of a sample of ETGs with the Integral Field Unit SAURON, led the ATLAS $^{3 \mathrm{D}}$ team to exclude a universal IMF, suggesting a trend, towards higher $\mathrm{M} / \mathrm{L}$, with increasing velocity dispersion (Cappellari et al., 2012). Such a result would be compatible with the constraints based on gravity-sensitive features. The other probe of the IMF, based on gravitational lensing, requires specific strong lenses where the line of sight to the background source lies very close to the centre of the lensing galaxy. Such a lens geometry is very sensitive to the projected mass 
within a small radius of the centre of the lensing galaxy. In these studies the contribution from dark matter is also expected to be subdominant. However, note that lensing masses are constrained within a cylinder along the line of sight. Studies based on this methodology have given consistent trends with the other probes, namely a heavier $\mathrm{M} / \mathrm{L}$ in the more massive ETGs (e.g. Posacki et al., 2015). However, some recent gravitational lenses have posed challenging constraints against this trend (Smith et al., 2015).

Figure 4 shows an outline of the three independent probes of the IMF in unresolved, passive stellar populations. Additional probes that would give an independent confirmation of this trend should target the high-mass end of the IMF through the analysis of star formation indicators such as NUV fluxes, recombination lines, or even FIR photometry in massive galaxies at high redshift. These probes will be fundamental to the analysis of the progenitors of high-mass ETGs.

\section{IMF and the physics of galaxy and star formation}

At present, there is no complete theory of star formation. There are many complexities underlying the multiple physical mechanisms at play during the transformation from gas into stars. Furthermore, these processes are relevant over a vast dynamical range in density; from the tenuous gas in the diffuse interstellar medium to the clumps formed in giant molecular clouds. No ab initio hydrodynamical simulation of star formation is capable of solving the problem, although state-of-the-art numerical simulations have greatly advanced our understanding of star formation (e.g. Bate et al., 2003). Variations in the IMF should be related to the way fragmentation operates during the last phases leading to star formation. Notice that the variations observed take place in massive ETGs. These galaxies are thought to form a large fraction of their stellar mass within an early and relatively short period of time (e.g. de La Rosa et al., 2011). Therefore, the physical conditions of the gas phase during their formation must be vastly different from the typical (nearby) star forming regions where resolved stellar analyses are performed. Nevertheless, it is still not known whether one should expect a significant variation of the IMF or not. Arguments based on turbulence in a highly supersonic medium suggest an enhanced fragmentation process, leading towards a bottom-heavy IMF (e.g. Padoan \& Nordlund, 2002; Chabrier et al., 2014). However, local observations of strongly star forming systems, i.e. scaled-down equivalents of the progenitors of massive ETGs - suggest a top-heavy IMF instead (i.e. an excess of high-mass stars (Gunawardhana et al., 2011). Such a confusing scenario is compounded by the fact that a simple model of galactic chemical enrichment robustly rules out a time-independent bottom-heavy IMF (Weidner et al., 2013). Therefore, if we take the evidence presented in this article at face value, we find that one should not only expect IMF variations among galaxies. Changes in the IMF during the formation process of a galaxy should also be present in the most massive systems. In this respect, the presence of radial gradients of the IMF within the same galaxy should support this scenario. However, we are still a long way from a complete picture of this essential piece of the cosmic puzzle of galaxy formation.

\section{References}

Bagnulo, S., Jehin, E., Ledoux, C., et al. 2003, The Messenger, 114, 10

Bate, M. R., Bonnell, I. A., \& Bromm, V. 2003, MNRAS, 339, 577

Bruzual, G., \& Charlot, S. 2003, MNRAS, 344, 1000

Cappellari, M., McDermid, R. M., Alatalo, K., et al. 2012, Nature, 484, 485

-. 2013, MNRAS, 432, 1862
Cenarro, A. J., Gorgas, J., Vazdekis, A., Cardiel, N., \& Peletier, R. F. 2003, MNRAS, 339, L12

Chabrier, G., Hennebelle, P., \& Charlot, S. 2014, ApJ, 796, 75

Conroy, C., \& van Dokkum, P. 2012a, ApJ, arXiv:1109.0007

Conroy, C., \& van Dokkum, P. G. 2012b, ApJ, arXiv:1205.6473

de La Rosa, I. G., La Barbera, F., Ferreras, I., \& de Carvalho, R. R. 2011, MNRAS, 418, L74
De Lucia, G., Springel, V., White, S. D. M., Croton, D., \& Kauffmann, G. 2006, MNRAS, 366, 499

Dotter, A., Chaboyer, B., Jevremović, D., et al. 2008, ApJS, 178, 89

Ferreras, I., La Barbera, F., de la Rosa, I. G., et al. 2013, MNRAS, 429, L15

Ferreras, I., \& Silk, J. 2000, ApJ, 532, 193

Gunawardhana, M. L. P., Hopkins, A. M., Sharp, R. G., et al. 2011, MNRAS, 415, 1647 
Kroupa, P., Weidner, C., PflammAltenburg, J., et al. 2013, The Stellar and Sub-Stellar Initial Mass Function of Simple and Composite Populations, ed. T. D. Oswalt \& G. Gilmore, 115

La Barbera, F., Ferreras, I., Vazdekis, A., et al. 2013, MNRAS, 433, 3017

La Barbera, F., Pasquali, A., Ferreras, I., et al. 2014, MNRAS, 445, 1977

Maraston, C. 2005, MNRAS, 362, 799

Martín-Navarro, I., Barbera, F. L., Vazdekis, A., Falcón-Barroso, J., \& Ferreras, I. 2015a, MNRAS, arXiv:1404.6533

Martín-Navarro, I., Vazdekis, A., La Barbera, F., et al. 2015b, ApJ, arXiv:1506.00638

Padoan, P., \& Nordlund, Å. 2002, ApJ, 576,870
Pietrinferni, A., Cassisi, S., Salaris, M., \& Castelli, F. 2006, ApJ, 642, 797

Posacki, S., Cappellari, M., Treu, T., Pellegrini, S., \& Ciotti, L. 2015, MNRAS, 446, 493

Saglia, R. P., Maraston, C., Thomas, D., Bender, R., \& Colless, M. 2002, ApJ, 579, L13

Salpeter, E. E. 1955, ApJ, 121, 161

Sánchez-Blázquez, P., Peletier, R. F., Jiménez-Vicente, J., et al. 2006, MNRAS, 371, 703

Smith, R. J., Lucey, J. R., \& Conroy, C. 2015, MNRAS, 449, 3441

Spiniello, C., Trager, S. C., \& Koopmans, L. V. E. 2015, ApJ, 803, 87

Spinrad, H. 1962, ApJ, 135, 715

Spinrad, H., \& Taylor, B. J. 1971, ApJS, 22, 445
Thomas, D., Maraston, C., Bender, R., $\&$ Mendes de Oliveira, C. 2005, ApJ, 621,673

Tinsley, B. M., \& Gunn, J. E. 1976, ApJ, 203, 52

Trager, S. C., Faber, S. M., Worthey, G., \& González, J. J. 2000, AJ, 119, 1645

van Dokkum, P. G., \& Conroy, C. 2010, Nature, 468, 940

Vazdekis, A., Ricciardelli, E., Cenarro, A. J., et al. 2012, MNRAS, 424, 157

Vazdekis, A., Coelho, P., Cassisi, S., et al. 2015, MNRAS, 449, 1177

Weidner, C., Ferreras, I., Vazdekis, A., \& La Barbera, F. 2013, MNRAS, 435, 2274

Worthey, G. 1994, ApJS, 95, 107 


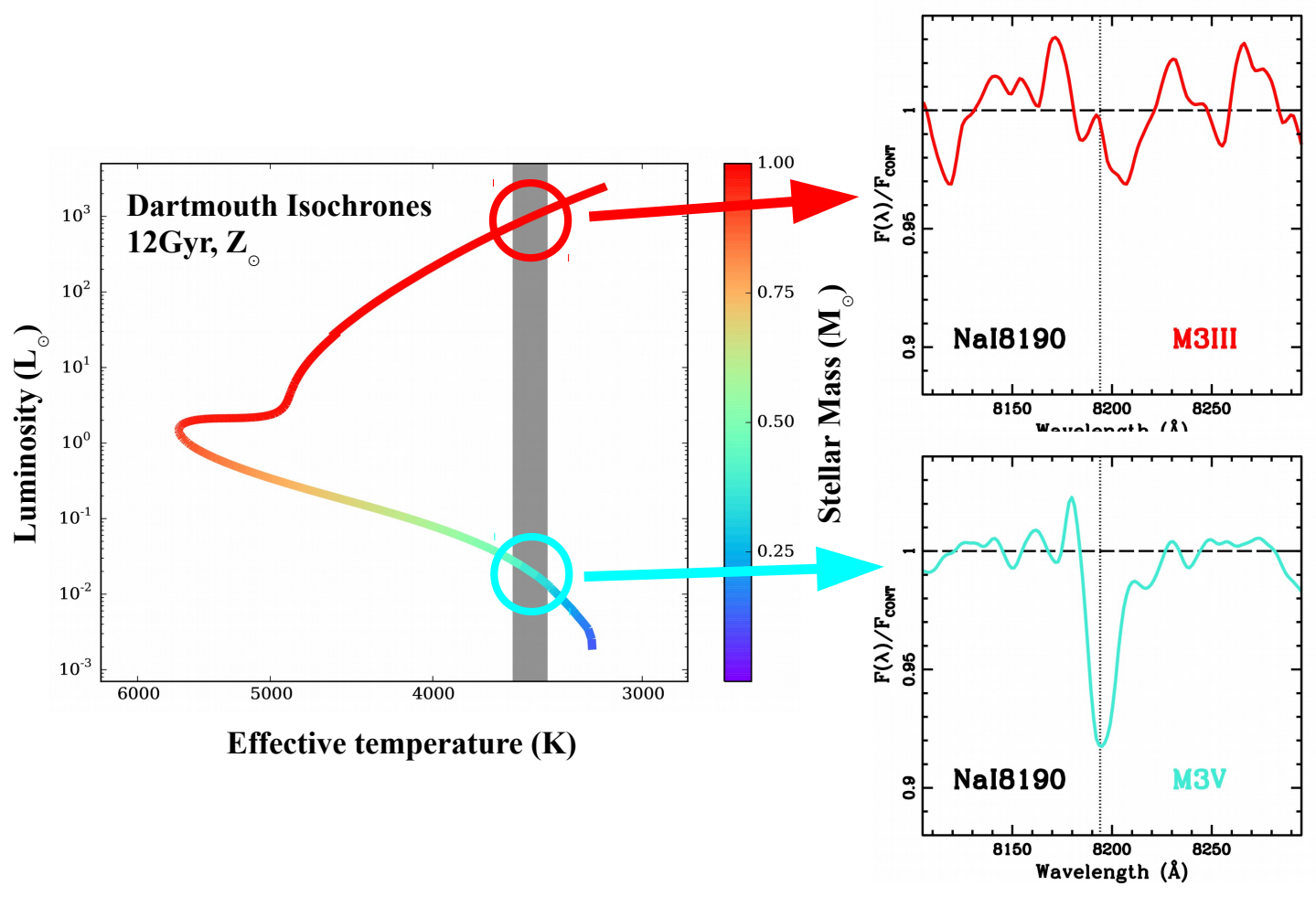

Figure 1: Schematic view of a gravity-sensitive feature and its application to constrain the IMF. The diagram on the left is a Hertzsprung-Russell diagram for an old $(12 \mathrm{Gyr})$ population with solar metallicity. The colour-coding corresponds to stellar mass. The vertical shaded area focuses on a cool surface temperature (spectral type M3), where two types of stars can be discerned: Giants (top) and main sequence, dwarf stars (bottom). The panels on the right show the characteristic spectrum of these two types of stars around the $8190 \AA$ NaI feature. Note the significant difference between the two. Therefore, a change in the IMF will result in a different relative contribution between these two types of stars in the spectral feature (Isochrone from the Dartmouth group, Dotter et al. 2008. Stellar spectra from the MILES database, Sánchez-Blázquez et al. 2006). 


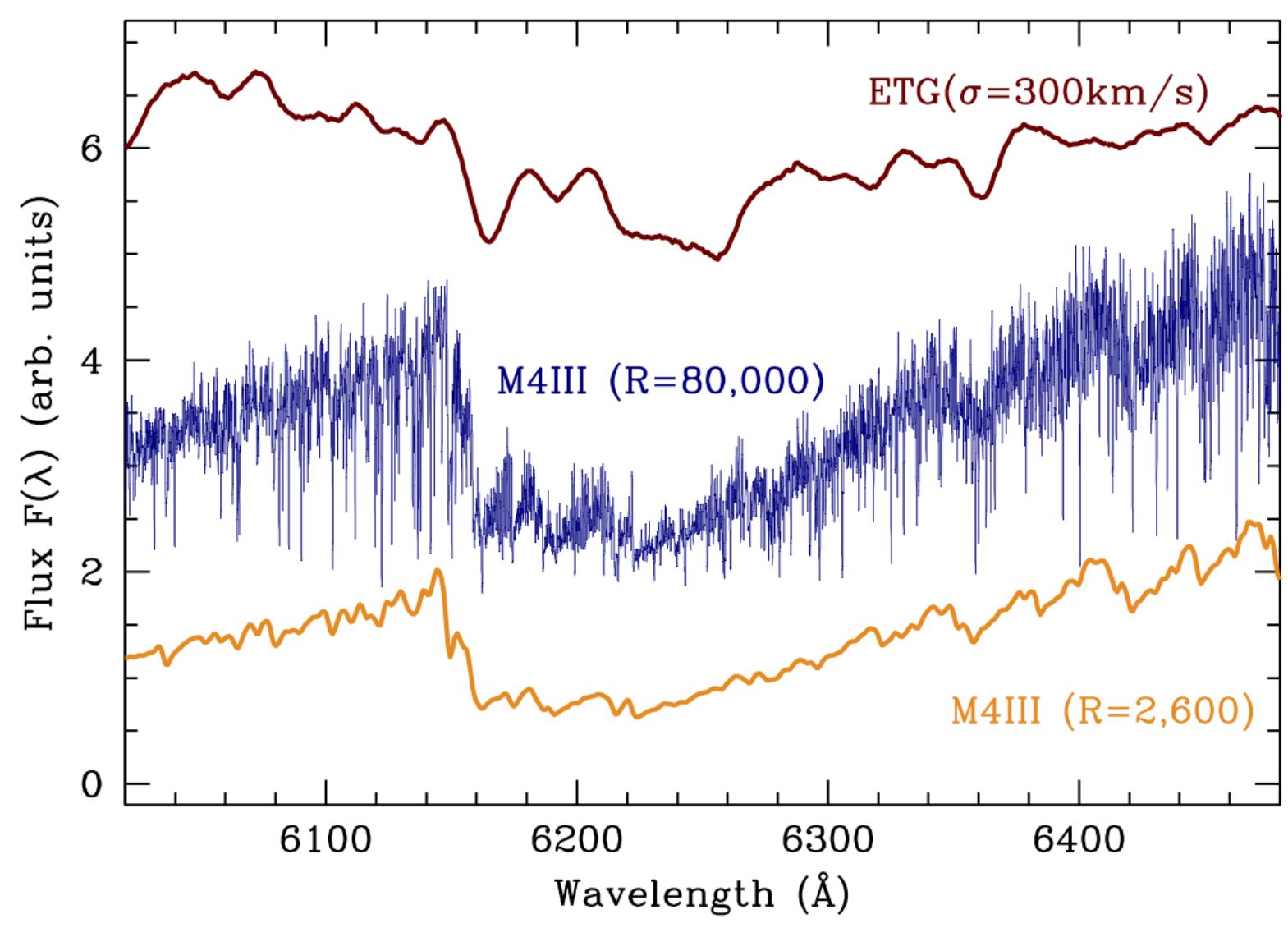

Figure 2: The difficulty in the interpretation of spectral line strengths in unresolved stellar populations is illustrated here, comparing a cool (M-type) giant star, at a high spectral resolution (blue, from the UVES POP library, Bagnulo et al. 2003), and the same star at the typical resolution used by population synthesis models (orange, from the MILES library, Sánchez-Blázquez et al. 2006). Note how many of the narrow absorption lines are blended and washed out in the process. Along with this complication, a galaxy (a typical case of a massive ETG is shown in red, from Ferreras et al. 2013) introduces an additional smoothing of the spectra becasue of the stellar velocity dispersion, in addition to a complex superposition of a large number of stars with different ages and chemical composition. This figure shows the spectral window where the gravity-sensitive index TiO2 $2_{\text {SDSS }}$ is defined (central bandpass 6189.6-6272.1 $\mathrm{A}$, La Barbera et al. 2013). 


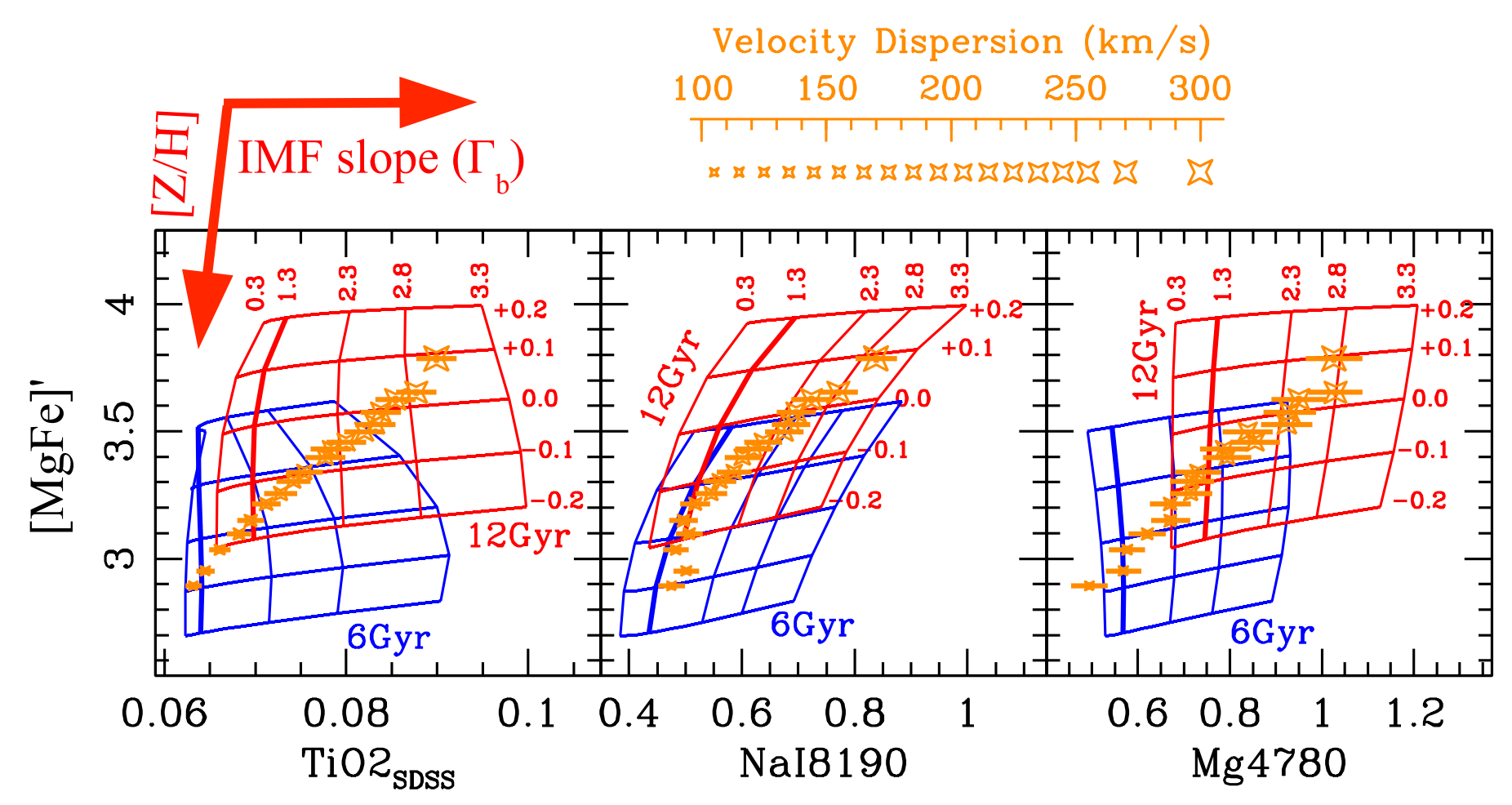

Figure 3: An example of the IMF-driven trends derived from spectroscopic line strengths. These diagrams plot a metallicity-sensitive index ([MgFe $\left.]^{\prime}\right)$ against three gravity-sensitive indices (from left to right, $\mathrm{TiO} 2_{\mathrm{SDSS}}$, NaI8190, and $\mathrm{Mg} 4780$ ). The orange 4-point star symbols correspond to observations from the Sloan Digital Sky Survey, stacking thousands of high-quality galaxy spectra with respect to stellar velocity dispersion, i.e. a rough proxy for the mass of the galaxy. The grids represent the derived indices from population synthesis models (Vazdekis et al., 2012), and are shown for a range of values of metallicity (labelled $[\mathrm{Z} / \mathrm{H}]$, measured as $\log \left(Z / Z_{\odot}\right)$, where $Z_{\odot}$ is the solar metallicity) and IMF slope - from a top-heavy $\Gamma_{b}=0.3$ to a bottom-heavy $\Gamma_{b}=3.3$. The Milky-Way equivalent corresponds to $\Gamma_{b}=1.3$, shown as a thick line in all grids. The age dependence is illustrated by showing two different grids, at $6 \mathrm{Gyr}$ (blue) and $12 \mathrm{Gyr}$ (red). Note that at the high-mass end (large stars, with velocity dispersion $\sim 300 \mathrm{~km} \mathrm{~s}{ }^{-1}$ ), the observations of all three gravity-sensitive indices require a bottom-heavy IMF, regardless of the other population parameters. 


\section{Three probes of the IMF in unresolved populations}

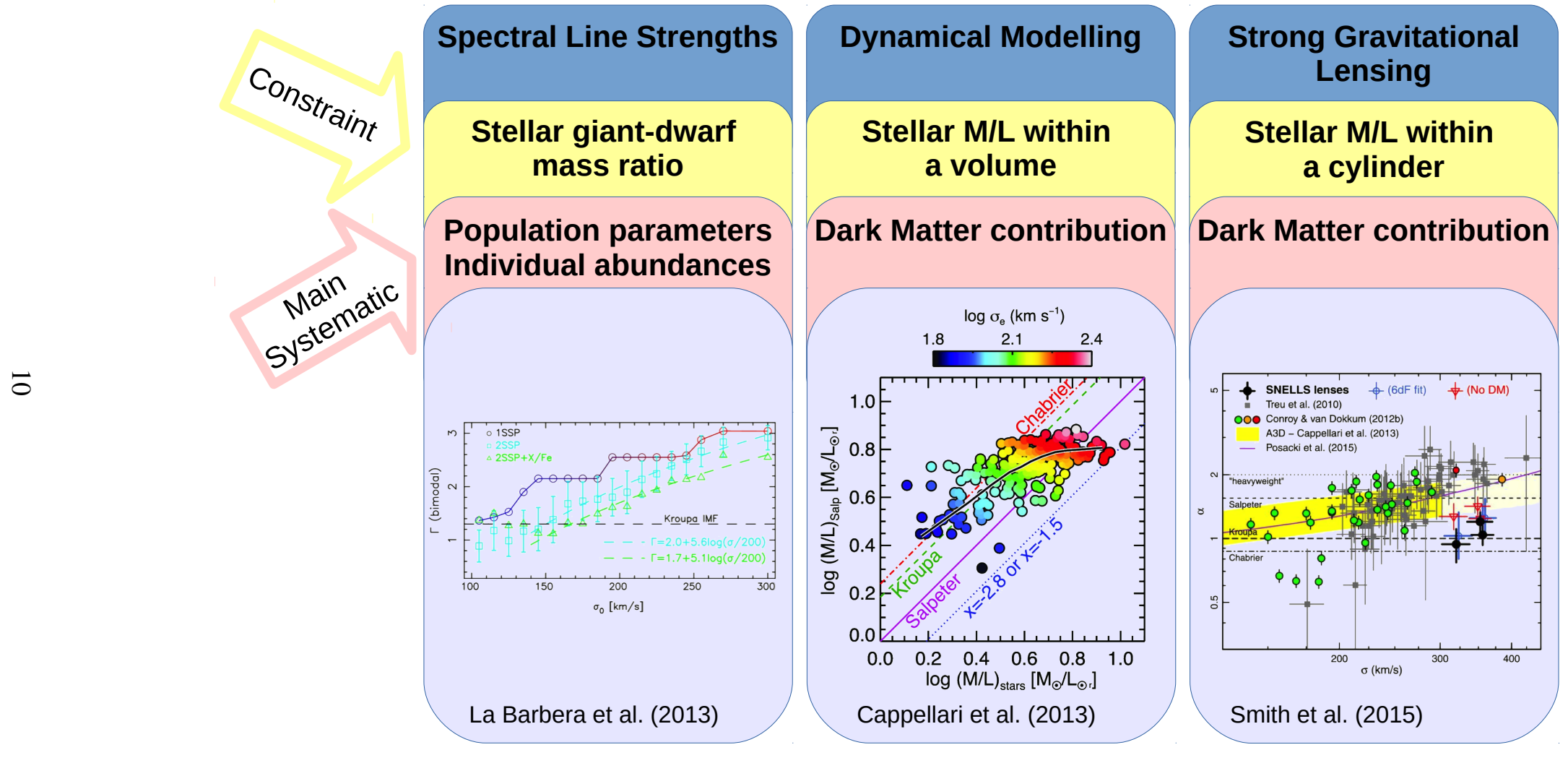

Figure 4: Three independent probes of the IMF of unresolved stellar populations in ETGs, including recent results based on these three methods. 